International Journal of Engineering \& Technology, $7(3.12)(2018) 1053-1055$
International Journal of Engineering \& Technology
SPC
Website: www.sciencepubco.com/index.php/IJET
Research paper

\title{
Safety based Virtual Positioning System for Ad-Hoc Wireless Network
}

\author{
Ganesh Gupta $^{1}$, Vivek Jaglan ${ }^{2}$, Ashok K Raghav ${ }^{3}$ \\ ${ }^{1}$ Research Scholar, ${ }^{2}$ Associate Professor, ${ }^{3}$ Professor, \\ ${ }_{1,2,3}^{3}$ Amity University Haryana \\ *Corresponding Author E-mail: ${ }^{1}$ ganeshgupta81@gmail.com \\ ${ }^{2}$ vjaglan@ggn.amity.edu, ${ }^{3}$ akraghav@ggn.amity.edu
}

\begin{abstract}
In the age of smart computing and advance global positioning system, it has been observed that day by day natural calamity affects our resources differently in regular way. One of the most important tasks is to deploy ability for rescue inside infrastructure-less network in more accident prone area. The security could be obtained through either automatic monitoring system or manually monitoring system by enabling several electronic means and sensors. Virtual positioning system is one of the most relevant computational areas where one can restrict unauthorized access while monitoring each one of single event more precisely. This paper tries to add more security feature for a given Ad-Hoc wireless network area by utilizing SVPS (Secure Virtual Positioning System). In addition to it our proposed SVBS will be more effective security system to avoid hazards.
\end{abstract}

Keywords: 2D;3D;AODV;CAD;Digital Aroma ;GIS; Virtual Modeling; etc.

\section{Introduction}

In recent era where development of computers and Information technology is on its extreme, people require to use computer based techniques for virtualization of the reality. To satisfy the people's expectation many areas of virtual geographic environment have been studied by scientist, the study of accident prone area has included so that the system might able to control and overcome with it when the hazardous situation occurs in future. Virtual infrastructure construction has been gain more attention and popularity recently.

As we all aware with the development of Geographic Information System, Today many institutions and individual firms have established their own virtual campus based on 2D GIS. We require a level of interpretation and imagination for understanding a twodimensional representation of the real geographical position. The capability of GIS is to produce a dynamic and attractive threedimensional map which is one of most practical benefits for our consideration [1].

Organization of paper included six main sections, first section introduces the recent era developments, Second section gives the view of existing concepts about Virtual digital Campus, Third section describe about 3D Geographical Information System, Fourth Section focus about safety concern in Ad-Hoc Wireless network, Fifth and Six tells about the proposed work methods and conclusion.

\section{Virtual Digital Campus}

Virtual digital campus is based on the network which realizes the environment, resources and their activities digitally.
We build a digital aroma around us to broaden the vertical and horizontal dimensions of the real campus, which can be measured on scale of time and space.

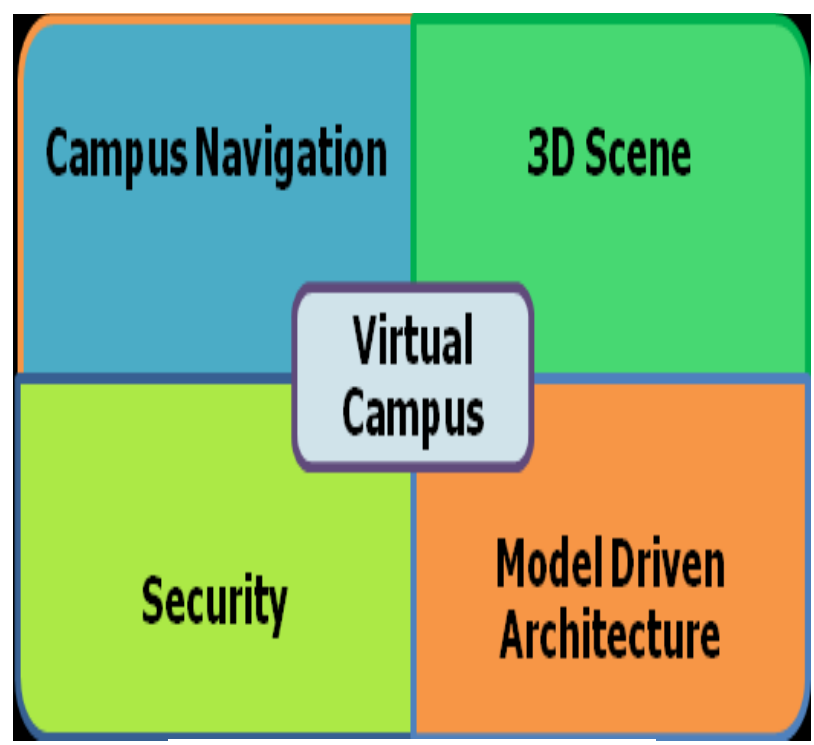

Fig. 1: The Structure of Virtual Campus[6]

Geographic Information system is an effective tool for processing special information, not only integrated in a unified platform for management but also it is unique geospatial data processing and analysis methods can develop various application modules, these application modules can provide campus development plan. 


\section{3D GIS}

As most of the virtual campus uses two dimensional GIS, which cannot meet the demands for precise positioning of real environment at the time of most critical requirement of security accurate positioning must be particularly important. Three Dimension (3D) technologies are used to simulate virtual campus network in building.

Comparing with 2D GIS and 3D GIS shows geospatial phenomenon to the users by dimensional modeling technology [2]. 3D GIS technology applied to the construction of virtual campus performance by modeling of real dimension. The diverse need of user such as 3D browsing, path query, analysis of results and attribute query could be fulfilled with help of virtual 3D GIS technology.

\section{Safety in Ad-Hoc Wireless Network}

\subsection{Safety Monitoring System:}

It is prominent need of any campus is to provide primary safety measures like first Aid and fire rescue equipments. The awareness could be one of the valid and effective tools for spreading information in society. As we know the student and intellectuals could play measure role for the same. We may proceed for secure channel with help of automatic safety monitoring equipment of firefighting and first aid services.

\subsection{Security inside Virtual Campus:}

To manage virtual campus physical security needs with Blackboard SMS, a complete, enterprise-class system designed specifically for the unique requirements of the general community that integrates access control, video surveillance, and monitoring capabilities.

\section{Proposed Precise Positioning System}

\subsection{Data Collection:}

First of all we have selected a different resolution and accuracy of data and 3D image according to the requirement. According to the building structure, environment and other scenes we included original and their virtual campus data. After getting the drawing from relevant departments added it with campus design CAD maps plan. Now we are ready to collect instant camera captured scene to obtain information digitally.

\subsection{Appling the Precise Positioning System for Existing Ad-Hoc Wireless Network:}

Let put the equipment inside one part of the ad-hoc network where anyone who wants to check the correctness of the system experimentally. We create visual simulation scenarios, roaming management system to create 3D image using 3D graphic image processing software to enhance ad-hoc network management and decision making analysis. We would establish the appropriate spatial database, and achieve query taking capability for desired orientation of location. At last after integrating all these elements and 3D GIS as shown in figure 2 the safe precise positioning system has been deployed.

\subsection{Result Analysis:}

We have used network simulator NS2 [8] to get the comparative result. With increasing the network size, we have compared AODV and SVBS packet delivery Ratio using simulator and the result shown in figure 3 .

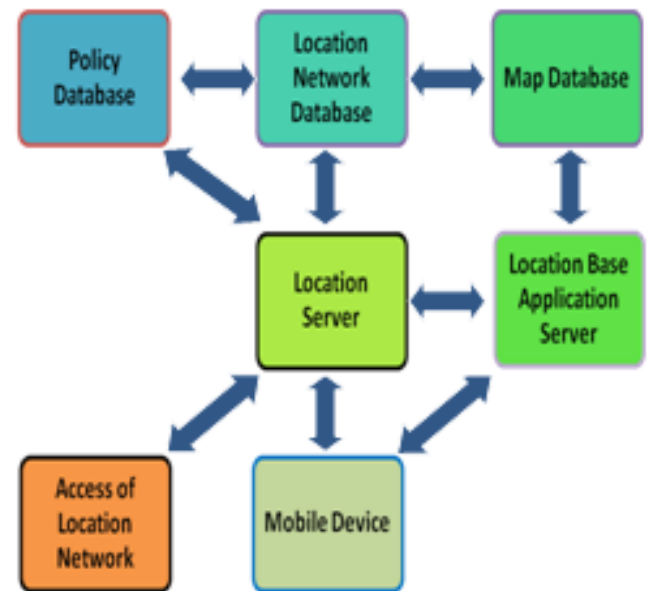

Fig. 2: Safe Precise Positioning System [6]

Assuming the malicious node in network the performance load graph increase in case of AODV however it is decreases for SVBS, which show the given system is safer than AODV.

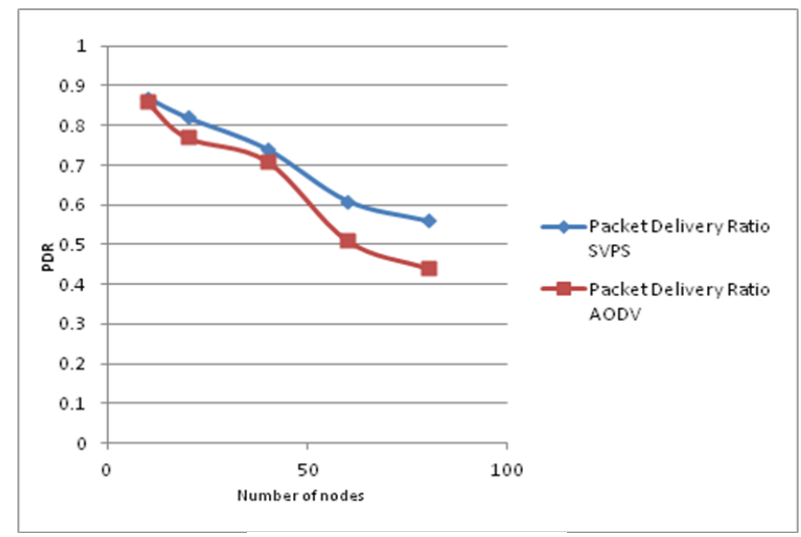

Fig. 3: Effect of netwok size

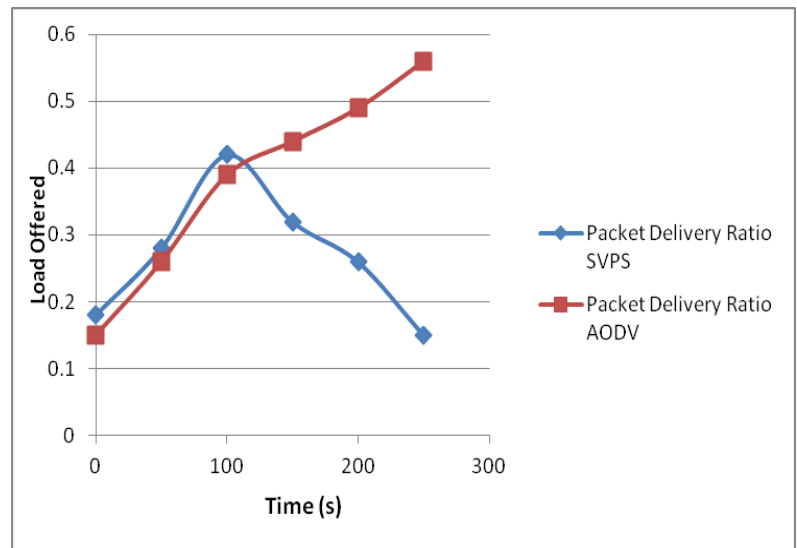

Fig. 4: Effect on network after Malicious node insertion

\section{Conclusion}

During the accidental incidence there is high demand of secure channels for the rescue process. Our work fulfils all the simulation based security measures to update alert in such incident. Deployment of precise target rescue equipment executed successfully. We can quickly react with add situation on basis of the simulation decisions. Hence it can greatly reduce the causality and other losses. 


\section{References}

[1] https://www.ordnancesurvey.co.uk/support/understandinggis/whatis-gis-data.html.

[2] Jiang Dongxing, Chen Huaichu, University Resource Planning Theory \& Practice. Education and Information Technology, 2005(9)

[3] Zhang Hui, Fu Yao. Scene Analysis based Interactive Navigation, Journal of Southeast University 2001, Vol. 31 (02).

[4] Li Long, Xiang Minghang, Liu Xianmei, The Construction and Cruise Engine of Virtual Campus Environment. Journal of Daqing Petroleum College, 2003(02).

[5] Ren Wu-ling, Feng Cheng-rong, Jiang Guo-xin, Precise positioning system of security failure in virtual campus, International Conference on Computation and Information Sciences, IEEE ,pp. 17211724. 2013

[6] G. Gupta, "Safety based Virtual Positioning System for existing infrastructure "JETIR, Vol. 5 Issue 5,pp-790-791 2018(5).

[7] Li Yahui, Yang Hongwa \& Xie Fei "A security routing protocol of Ad-Hoc Network based on node trust evaluation"ICINS-2014, IEEE Explore, 2015

[8] The NS Manual Http:// www.isi.edu/nsnam/ns/ns-documentation 